\title{
A Numerical Study of Crown Forest Fires Behaviour
}

\author{
Valeriy Perminov \\ Tomsk Polytechnic University \\ Tomsk, Russia \\ valerperminov@gmail.com
}

\begin{abstract}
The theoretical investigation of the problems of large forest fires initiation and spread was carried out in this paper. Mathematical model of forest fire was based on an analysis of experimental data and using concept and methods from reactive media mechanics. The research was based on numerical solution of three dimensional Reynolds equations. The boundary-value problem is solved numerically using the method of splitting according to physical processes. A discrete analogue for the system of equations was obtained by means of the control volume method. The developed numerical model of forest fire initiation and spreading would make it possible to obtain a detailed picture of the variation in the velocity, temperature and chemical species concentration fields with time. The results of calculation give an opportunity to describe conditions of crown forest fires initiation and spread at different properties of forests and meteorological conditions. Forest fires contours and their sizes depend on crown properties: bulk density, moisture content of forest fuel, distribution of combustion sources and etc.
\end{abstract}

Keywords-forest fire; combustion; turbulence; control volume; discrete analogue

\section{INTRODUCTION}

Analysis of the characteristics of forest fires reveals their dependence on the specific conditions under which the experiments were conducted, and their insufficient accuracy, which results from the fact that under actual conditions it is impossible to control the meteorological situation and the homogeneity of forest fuel layer. For this reason the physical modeling of forest fires must be combined with mathematical experiments using numerical methods and computers. The object of study in this case is not nature, but a mathematical model of a forest fire, which usually consists of a set of partial differential equations with appropriate initial and boundary conditions. Integral or functional equations can be used instead of differential. The deduction of a system of equations, and boundary and initial conditions, and the subsequent solution of the corresponding mathematical problem is usually called mathematical modeling of forest fires. Mathematical modeling possesses a number of advantages over physical experimentation, for example, ecological safety and greater economic efficiency. One of the objectives of these studies is the improvement of knowledge on the fundamental physical mechanisms that control forest fire initiation and spread. Considering that, natural investigations of these problems are merely impossible, methods of mathematical modeling are urgent. Mathematical model of forest fire was based on an analysis of known experimental data and using concept and methods from reactive media mechanics. A great deal of work has been done on the theoretical problem of how forest fire initiation. The first explanation of this process was given by Van Wagner [1]. The theory proposed there depends on three simple crown properties: crown base height, bulk density and moisture content of forest fuel. Also crown fire initiation and hazard have been studied and modeled in [2-8]. Besides the discussion of the problem of modeling forest fires is provided by Grishin and etc. [9-12]). In particular, a mathematical model of forest fires was obtained by Grishin [10] based on an analysis of known and original experimental data $[9,13]$, and using concepts and methods from reactive media mechanics. The physical two-phase models used in [14-15] may be considered as a continuation and extension of the formulation proposed by Grishin and Perminov [10-12]. However, the investigation of crown fires initiation has been limited mainly to cases studied of forest fires initiation as the result of transition of surface fires into crown forest fires. The present mathematical model and results of calculation in this paper are used to understand and to predict possible forest fires initiation and spread.

\section{PHYSICAL AND MATHEMATICAL FORMULATION}

The basic assumptions adopted during the deduction of equations, and boundary and initial conditions: 1) the forest represents a multi-phase, multistoried, spatially heterogeneous medium; 2) in the fire zone the forest is a porous-dispersed, two-temperature, single-velocity, reactive medium; 3) the forest canopy is supposed to be non - deformed medium (trunks, large branches, small twigs and needles), affects only the magnitude of the force of resistance in the equation of conservation of momentum in the gas phase, i.e., the medium is assumed to be quasi-solid (almost non-deformable during wind gusts); 4) let there be a so-called "ventilated" forest massif, in which the volume of fractions of condensed forest fuel phases, consisting of dry organic matter, water in liquid state, solid pyrolysis products, and ash, can be neglected compared to the volume fraction of gas phase (components of air and gaseous pyrolysis products); 5) the flow has a developed turbulent nature and molecular transfer is neglected; 6) gaseous phase density doesn't depend on the pressure because of the low velocities of the flow in comparison with the velocity of the sound. In the forest there is combustion source, which is defined as area of high temperature and source from the masses of the products of pyrolysis and combustion. Let the coordinate reference point $x_{1}, x_{2}, x_{3}=0$ be situated at the height of the roughness level, axis $0 x_{1}$ directed parallel to the Earth's surface to the right in the direction of the unperturbed wind speed, axis 
$0 x_{2}$ directed perpendicular to $0 x_{1}$ and axis $0 x_{3}$ directed upward (Fig. 1).

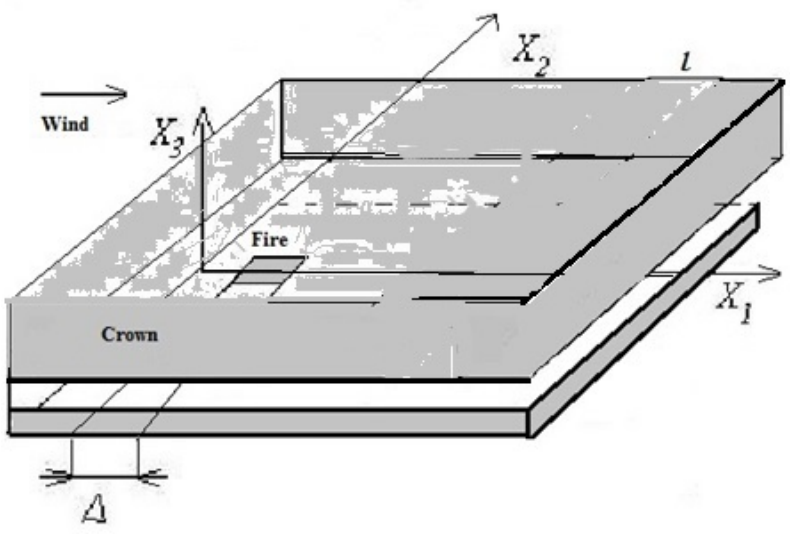

Fig.1

Because of the horizontal sizes of forest massif more than height of forest, system of equations of general mathematical model of forest fire [9] was integrated between the limits from height of the roughness level - 0 to $h$. The problem formulated above is reduced to a solution of the following system of equation:

$\frac{\partial \rho}{\partial t}+\frac{\partial}{\partial x_{j}}\left(\rho v_{j}\right)=Q-\left(\dot{m}^{-}-\dot{m}^{+}\right) / h, j=1,2 ;$

$\rho \frac{d v_{i}}{d t}=-\frac{\partial p}{\partial x_{i}}+\frac{\partial}{\partial x_{j}}\left(-\rho{\overline{v_{i}^{\prime}}}_{j}^{\prime}\right)-\rho s c_{d} v_{i}|\vec{v}|-$

$\rho g_{i}-Q v_{i}+\left(\tau_{i}^{-}-\tau_{i}^{+}\right) / h, i=1,2,3$

$\rho c_{p} \frac{d T}{d t}=\frac{\partial}{\partial x_{j}}\left(-\rho c_{p} \overline{\left.v_{j}^{\prime} T^{\prime}\right)}+q_{5} R_{5}+\right.$

$+k_{g}\left(c U_{R}-4 \sigma T^{4}\right)-\alpha_{v}\left(T-T_{s}\right)-\left(q_{T}^{-}-q_{T}^{+}\right) / h ;$

$\rho \frac{d c_{\alpha}}{d t}=\frac{\partial}{\partial x_{j}}\left(-\rho \overline{v_{j}^{\prime} c_{\alpha}^{\prime}}\right)+R_{5 \alpha}-Q c_{\alpha}+$

$\left(J_{\alpha}^{-}-J_{\alpha}^{+}\right) / h, \alpha=1,3$;

$\frac{\partial}{\partial x_{j}}\left(\frac{c}{3 k} \frac{\partial U_{R}}{\partial x_{j}}\right)-k c U_{R}+4 k_{g} \sigma T^{4}+4 k_{S} \sigma T_{S}^{4}+$

$$
+\left(q_{R}^{-}-q_{R}^{+}\right) / h=0
$$

$\sum_{i=1}^{4} \rho_{i} c_{p i} \varphi_{i} \frac{\partial T_{S}}{\partial t}=q_{3} R_{3}-q_{2} R_{2}+$

$+k_{S}\left(c U_{R}-4 \sigma T_{S}^{4}\right)+\alpha_{V}\left(T-T_{S}\right)$;

$\rho_{1} \frac{\partial \varphi_{1}}{\partial t}=-R_{1}, \rho_{2} \frac{\partial \varphi_{2}}{\partial t}=-R_{2}$,

$\rho_{3} \frac{\partial \varphi_{3}}{\partial t}=\alpha_{C} R_{1}-\frac{M_{C}}{M_{1}} R_{3}, \rho_{4} \frac{\partial \varphi_{4}}{\partial t}=0 ;$ $\sum_{\alpha=1}^{3} c_{\alpha}=1, P_{e}=\rho R T \sum_{\alpha=1}^{3} \frac{c_{\alpha}}{M_{\alpha}}, \vec{v}=\left(v_{1}, v_{2}, v_{3}\right)$,

$\vec{g}=(0,0, g)$.

The system of equations (1)-(8) must be solved taking into account the initial and boundary conditions:

$$
\begin{aligned}
& t=0: v_{1}=0, v_{2}=0, v_{3}=0, T=T_{e}, T_{S}=T_{e} \text {, } \\
& c_{\alpha}=c_{\alpha e}, \varphi_{i}=\varphi_{i e} ; \\
& x_{1}=0: v_{1}=V_{e}\left(x_{3}\right), v_{2}=0, v_{3}=0, T=T_{e} \text {, } \\
& c_{\alpha}=c_{\alpha e},-\frac{c}{3 k} \frac{\partial U_{R}}{\partial x_{1}}+c U_{R} / 2=0 . \\
& x_{1}=x_{1 e}: \frac{\partial v_{1}}{\partial x_{1}}=0, \frac{\partial v_{2}}{\partial x_{1}}=0, \frac{\partial v_{3}}{\partial x_{1}}=0, \frac{\partial T}{\partial x_{1}}=0 \text {, } \\
& \frac{\partial c_{\alpha}}{\partial x_{1}}=0,-\frac{c}{3 k} \frac{\partial U_{R}}{\partial x_{1}}+c U_{R} / 2=0, \\
& x_{2}=0: \frac{\partial v_{1}}{\partial x_{2}}=0, \frac{\partial v_{2}}{\partial x_{2}}=0, \frac{\partial v_{3}}{\partial x_{2}}=0, \frac{\partial T}{\partial x_{2}}=0 \text {, } \\
& \frac{\partial c_{\alpha}}{\partial x_{2}}=0,-\frac{c}{3 k} \frac{\partial U_{R}}{\partial x_{2}}+c U_{R} / 2=0 \text {; } \\
& x_{2}=x_{2 e}: \frac{\partial v_{1}}{\partial x_{2}}=0, \frac{\partial v_{2}}{\partial x_{2}}=0, \frac{\partial v_{3}}{\partial x_{2}}=0, \frac{\partial T}{\partial x_{2}}=0 \\
& \frac{\partial c_{\alpha}}{\partial x_{2}}=0, \frac{c}{3 k} \frac{\partial U_{R}}{\partial x_{2}}+c U_{R} / 2=0, \\
& x_{2}=x_{2 e}: \frac{\partial v_{1}}{\partial x_{2}}=0, v_{2}=0, \frac{\partial v_{3}}{\partial x_{2}}=0, \frac{\partial T}{\partial x_{2}}=0 \text {, } \\
& \frac{\partial c_{\alpha}}{\partial x_{2}}=0, \frac{\partial U_{R}}{\partial x_{2}}=0 \\
& x_{3}=0: v_{1}=0, v_{2}=0,\left(\rho v_{3}\right)=h_{0} \overline{\dot{m}} \text {, } \\
& -\rho D_{t} \frac{\partial c_{\alpha}}{\partial x_{3}}+\rho v_{3} c_{\alpha}=h_{0} \bar{R}_{5 \alpha}, \\
& T=\left\{\begin{array}{c}
T_{e}+\left(T_{0}-T_{e}\right) \frac{t}{t_{0}} \exp \left\{-[r / \Delta]^{2}\right\}, t \leq t_{0}, \\
T_{e}+\left(T_{0}-T_{e}\right) \exp \left\{-\left[\left(r-r_{f}\right) / \Delta\right]^{2}\right\}, t>t_{0},
\end{array}\right. \\
& -\frac{c}{3 k} \frac{\partial U_{R}}{\partial x_{3}}=\frac{\varepsilon}{2(2-\varepsilon)}\left(4 \sigma T_{S}^{4}-c U_{R}\right) \text {; } \\
& x_{3}=x_{3 e}: \frac{\partial v_{1}}{\partial x_{3}}=0, \frac{\partial v_{2}}{\partial x_{3}}=0, \frac{\partial v_{3}}{\partial x_{3}}=0, \frac{\partial T}{\partial x_{3}}=0 \text {, } \\
& \frac{\partial c_{\alpha}}{\partial x_{3}}=0, \frac{c}{3 k} \frac{\partial U_{R}}{\partial x_{3}}+c U_{R} / 2=0 .
\end{aligned}
$$


Here and above $\frac{\mathrm{d}}{\mathrm{d} t}$ is the symbol of the total (substantial) derivative; $\alpha_{V}$ is the coefficient of phase exchange; $\rho$ - density of gas - dispersed phase, $t$ is time; $v_{i}$ - the velocity components; $T, T_{S}$, - temperatures of gas and solid phases, $U_{R}$ - density of radiation energy, $k$ - coefficient of radiation attenuation, $P$ - pressure; $c_{p}$ - constant pressure specific heat of the gas phase, $c_{p i}, \rho_{i}, \varphi_{\mathrm{i}}$ - specific heat, density and volume of fraction of condensed phase (1 - dry organic substance, 2 moisture, 3 - condensed pyrolysis products, 4 - mineral part of forest fuel), $R_{i}$ - the mass rates of chemical reactions, $q_{i}-$ thermal effects of chemical reactions; $k_{g}, k_{S}$ - radiation absorption coefficients for gas and condensed phases; $T_{e}$ - the ambient temperature; $c_{\alpha}$ - mass concentrations of $\alpha$ component of gas - dispersed medium, index $\alpha=1,2,3$, where 1 corresponds to the density of oxygen, 2 - to gas products of pyrolysis, 3 - to carbon dioxide and inert components of air; $R$ - universal gas constant; $M_{\alpha}, M_{C}$, and $M$ molecular mass of $\alpha$ -components of the gas phase, carbon and air mixture; $g$ is the gravity acceleration; $c_{d}$ is an empirical coefficient of the resistance of the vegetation, $s$ is the specific surface of the forest fuel in the given forest stratum. In system of equations (2)-(8) are introduced the next designations:

$$
\dot{m}=\rho v_{3}, \tau_{i}=-\rho \overline{v_{i}^{\prime} v_{3}^{\prime}}, J_{\alpha}=-\rho \overline{v_{3}^{\prime} c_{\alpha}^{\prime}}, J_{T}=-\rho \overline{v_{3}^{\prime} T^{\prime}}
$$

Upper indexes “+” and “-” designate values of functions at $x_{3}=h$ and $x_{3}=0$ correspondingly. It is assumed that heat and mass exchange of fire front and boundary layer of atmosphere are governed by Newton law and written using the formulas:

$$
\begin{aligned}
& \left(q_{T}^{-}-q_{T}^{+}\right) / h=-\alpha\left(T-T_{e}\right) / h, \\
& \left(J_{\alpha}^{-}-J_{\alpha}^{+}\right) / h=-\alpha\left(c-c_{\alpha e}\right) / h c_{p} .
\end{aligned}
$$

To define source terms which characterize inflow (outflow of mass) in a volume unit of the gas-dispersed phase, the following formulae were used for the rate of formulation of the gas-dispersed mixture $\dot{m}$, outflow of oxygen $R_{51}$, changing carbon monoxide $R_{52}$.

$$
\begin{aligned}
& Q=\left(1-\alpha_{c}\right) R_{1}+R_{2}+\frac{M_{c}}{M_{1}} R_{3}, R_{51}=-R_{3}-\frac{M_{1}}{2 M_{2}} R_{5}, \\
& R_{52}=v_{g}\left(1-\alpha_{c}\right) R_{1}-R_{5}, R_{53}=0 .
\end{aligned}
$$

Here $v_{\mathrm{g}}-$ mass fraction of gas combustible products of pyrolysis. Reaction rates of these various contributions (pyrolysis, evaporation, combustion of coke and volatile combustible products of pyrolysis) are approximated by Arrhenius laws whose parameters (pre-exponential constant $k_{i}$ and activation energy $E_{i}$ ) are evaluated using data for mathematical models $[9,11]$.

$$
\begin{aligned}
& R_{1}=k_{1} \rho_{1} \varphi_{1} \exp \left(-\frac{E_{1}}{R T_{s}}\right), R_{2}=k_{2} \rho_{2} \varphi_{2} T_{s}^{-0.5} \exp \left(-\frac{E_{2}}{R T_{s}}\right), \\
& R_{3}=k_{3} \rho \varphi_{3} s_{\sigma} c_{1} \exp \left(-\frac{E_{3}}{R T_{s}}\right), \\
& R_{5}=k_{5} M_{2}\left(\frac{c_{1} M}{M_{1}}\right)^{0.25} \frac{C_{2} M}{M_{2}} T^{-2.25} \exp \left(-\frac{E_{5}}{R T}\right) .
\end{aligned}
$$

The initial values for volume of fractions of condensed phases are determined using the expressions:

$$
\varphi_{1 e}=\frac{d\left(1-v_{z}\right)}{\rho_{1}}, \varphi_{2 e}=\frac{W d}{\rho_{2}}, \varphi_{3 e}=\frac{\alpha_{c} \varphi_{1 e} \rho_{1}}{\rho_{3}}
$$

where $d$-bulk density for surface layer, $v_{z}$ - coefficient of ashes of forest fuel, $W$ - forest fuel moisture content.

It is supposed that the optical properties of a medium are independent of radiation wavelength (the assumption that the medium is "grey"), and the so-called diffusion approximation for radiation flux density were used for a mathematical description of radiation transport during forest fires.

To close the system (1)-(8), the components of the tensor of turbulent stresses, and the turbulent heat and mass fluxes are determined using the local-equilibrium model of turbulence (Grishin, [9]). The system of equations (1)-(8) contains terms associated with turbulent diffusion, thermal conduction, and convection, and needs to be closed. The components of the tensor of turbulent stresses $\rho \overline{v_{i}^{\prime} v_{j}^{\prime}}$, as well as the turbulent fluxes of heat and mass $\overline{\rho v_{j}^{\prime} c_{p} T^{\prime}}, \overline{\rho v_{j}^{\prime} C_{\alpha}^{\prime}}$ are written in terms of the gradients of the average flow properties using the formulas

$$
\begin{gathered}
-\rho \overline{v_{i}^{\prime} v_{j}^{\prime}}=\mu_{t}\left(\frac{\partial v_{i}}{\partial x_{j}}+\frac{\partial v_{j}}{\partial x_{i}}\right)-\frac{2}{3} K \delta_{i j}, \\
-\rho \overline{v_{j} c_{p} T^{\prime}}=\lambda_{t} \frac{\partial T}{\partial x_{j}},-\rho \overline{v_{j} c_{\alpha}^{\prime}}=\rho D_{t} \frac{\partial c_{\alpha}}{\partial x_{j}}, \\
\lambda_{t}=\mu_{t} c_{p} / \operatorname{Pr}_{t}, \rho D_{t}=\mu_{t} / S c_{t}, \mu_{t}=c_{\mu} \rho K^{2} / \varepsilon,
\end{gathered}
$$

where $\mu_{t}, \lambda_{t}, D_{t}$ are the coefficients of turbulent viscosity, thermal conductivity, and diffusion, respectively; $P r_{t}, S c_{t}$ are the turbulent Prandtl and Schmidt numbers, which were assumed to be equal to 1 . In dimensional form, the coefficient of dynamic turbulent viscosity is determined using local equilibrium model of turbulence [9]. The thermodynamic, thermophysical and structural characteristics correspond to the forest fuels in the canopy of a different (for example pine $[9,11,13])$ type of forest. The system of equations (1)-(8) must be solved taking into account the initial and boundary conditions. The thermodynamic, thermophysical and structural characteristics correspond to the forest fuels in the canopy of a different type of forest; for example, pine forest (Grishin, Perminov [11]). 


\section{NUMERICAL METHODS AND RESULTS}

The boundary-value problem (1)-(15) is solved numerically using the method of splitting according to physical processes [11]. In the first stage, the hydrodynamic pattern of flow and distribution of scalar functions was calculated. The system of ordinary differential equations of chemical kinetics obtained as a result of splitting was then integrated. A discrete analogue was obtained by means of the control volume method using the SIMPLE like algorithm [16]. The accuracy of the program was checked by the method of inserted analytical solutions. Analytical expressions for the unknown functions were substituted in (1)-(8) and the closure of the equations were calculated. This was then treated as the source in each equation. Next, with the aid of the algorithm described above, the values of the functions used were inferred with an accuracy of not less than $1 \%$. The effect of the dimensions of the control volumes on the solution was studied by diminishing them. The time step was selected automatically.

Fields of temperature, velocity, component mass fractions, and volume fractions of phases were obtained numerically. The distribution of basic functions shows that the process of crown forest fire initiation goes through the next stages. The first stage is related to increasing maximum temperature in the fire source. At this process stage the fire source a thermal wind is formed a zone of heated forest fire pyrolysis products which are mixed with air, float up and penetrate into the crowns of trees. As a result, forest fuels in the tree crowns are heated, moisture evaporates and gaseous and dispersed pyrolysis products are generated. Ignition of gaseous pyrolysis products of the ground cover occurs at the next stage, and that of gaseous pyrolysis products in the forest canopy occurs at the last stage. As a result of heating of forest fuel elements of crown, moisture evaporates, and pyrolysis occurs accompanied by the release of gaseous products, which then ignite and burn away in the forest canopy. At the moment of ignition the gas combustible products of pyrolysis burns away, and the concentration of oxygen is rapidly reduced. The temperatures of both phases reach a maximum value at the point of ignition. The ignition processes is of a gas - phase nature. The distribution of temperature, concentrations of gas products of pyrolysis and oxygen in the forest fire front are presented in the Figure 2. It is seen that the combustion wave looks like as a soliton. The oxygen concentration drops to near zero in front of a fire. It is consumed in the combustion of the pyrolysis products, the concentration of which is reached their maximum before the maximum of temperature.

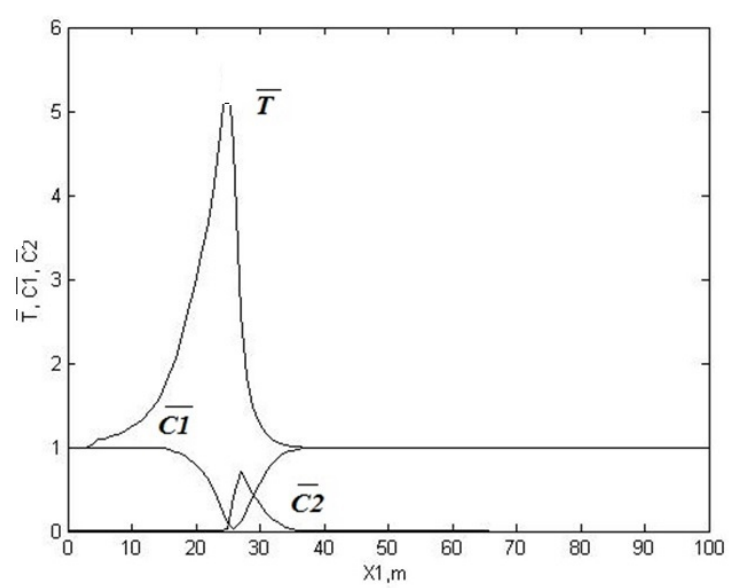

Fig. 2. The distribution of temperature $\bar{T}\left(\bar{T}=T / T_{e}, T_{e}=300 \mathrm{~K}\right)$, concentrations of gas products of pyrolysis $\bar{c}_{2}\left(\bar{c}_{2}=c_{2} / C_{1 e}, C_{1 e}=0.23\right)$ and oxygen $\bar{C}_{1}\left(\bar{c}_{1}=c_{1} / c_{1 e}\right)$ in forest fire front.

Figures 3 present the distribution for different instants of time for temperature a) $\bar{T}\left(\bar{T}=T / T_{e}, T_{e}=300 K\right)(1-1.5,2-2 ., 3$ $-2.6,4-3,5-3.5,6-4$.) for gas phase, b) oxygen $\bar{C}_{1}(1-$ $0.1,2-0.5,3-0.6,4-0.7,5-0.8,6-0.9)$, c) volatile combustible products of pyrolysis $\bar{C}_{2}$ concentrations $(1-1$., 2- 0.1, 3 - 0.05, 4 - 0.01) $\left(\bar{c}_{\alpha}=c_{\alpha} / c_{1 e}, c_{1 e}=0.23\right)$ and volume fraction of dry organic materials $\left.\overline{\varphi_{1}}\left(\overline{\varphi_{1}}=\varphi_{1} / \varphi_{1 e}\right), \mathrm{d}\right)$ for different instants of time. The isotherms are moved in the forest canopy and deformed by the action of wind (Figure 3a). Similarly, the fields of component concentrations oxygen (Figure 3b) are deformed. It is concluded that the forest fire begins to spread. The results of calculation give an opportunity to evaluate the size of burned square of forest (Fig.3d). Figure $3 . \mathrm{Ve}=3 \mathrm{~m} / \mathrm{s} ; \mathrm{z}=0.2 \mathrm{~kg} / \mathrm{m}^{3}, \mathrm{~W}=0.6$; I-5s, II $18 \mathrm{~s}$, III- 27s. The results of calculation show that the increase in wind speed has not led to a significant expansion of fire front (Figures 3-5).
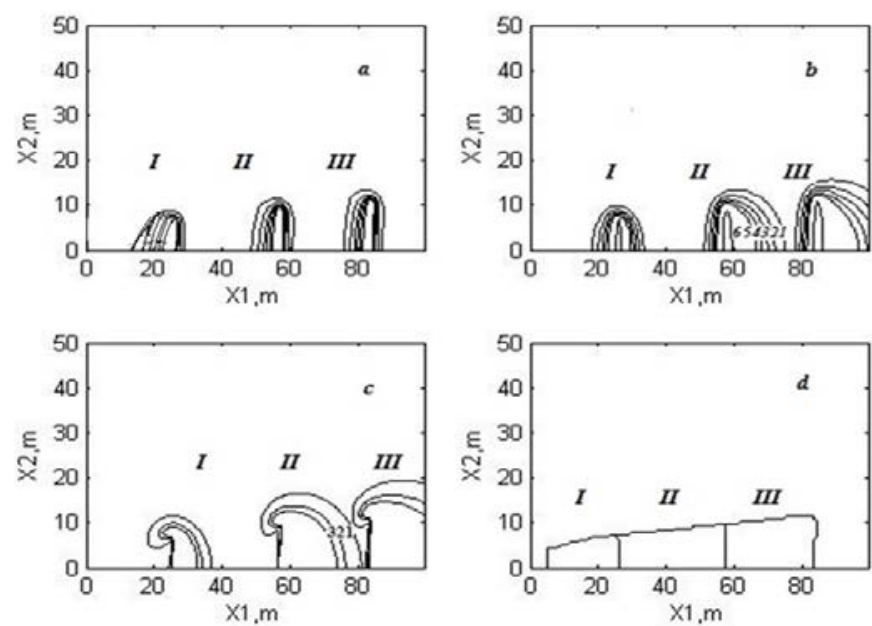

Fig. 4. $V e=5 \mathrm{~m} / \mathrm{s} ; \mathrm{z}=0.2 \mathrm{~kg} / \mathrm{m}^{3}, \mathrm{~W}=0.6 ; \mathrm{I}-5 \mathrm{~s}, \mathrm{II}-18 \mathrm{~s}, \mathrm{III}-24 \mathrm{~s}$. 
The most significant impact on the extension of the front crown fires are influenced during the change of the bulk and the moisture content of forest combustible materials. With increasing supply from 0.2 to 0.6 (Figures $4,8,9$ ) the width of the front fire increases more than two times. And by increasing the moisture content to 0.7 (Figures $4,6,7$ ) the width of the combustion front is practically unchanged.
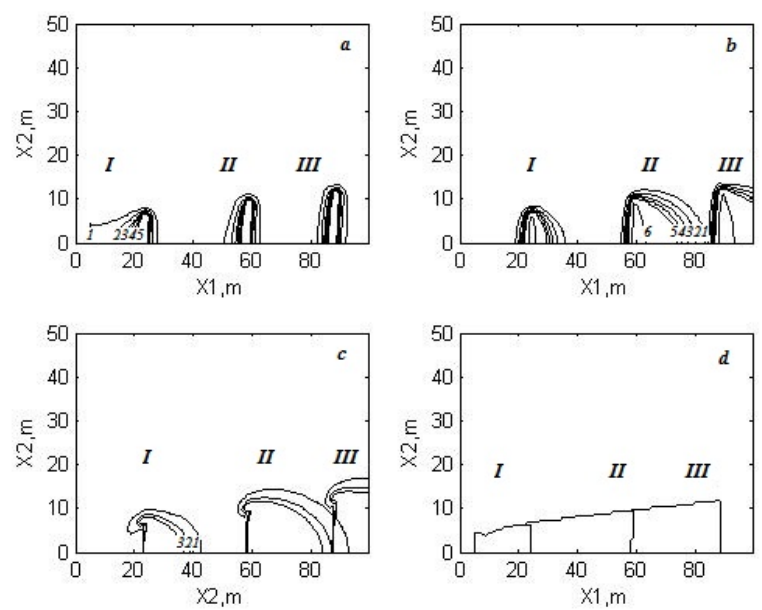

Fig. 5. Ve=10 m/s; z=0.2 kg/m³,$W=0.6$; I-2.5 s, II -10.5 s, III -14 s.
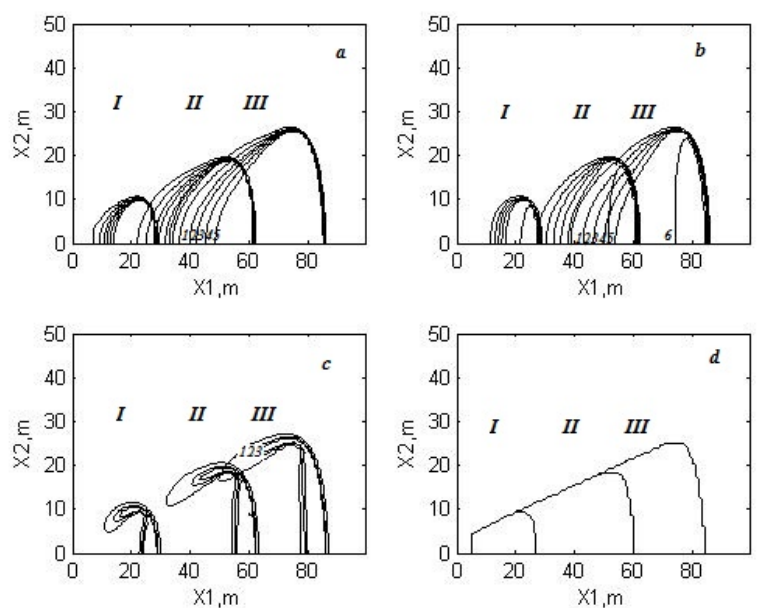

Fig. 6. $V_{\mathrm{e}}=5 \mathrm{~m} / \mathrm{s} ; \mathrm{z}=0.2 \mathrm{~kg} / \mathrm{m}^{3}, W=0.4$.

This study is conducted to study the behavior of crown forest fires. Based on these data we can estimate the minimum size of the fire break Based on these data we can estimate the minimum size of fire-breaks, with which you can stop the spread of the flame front.
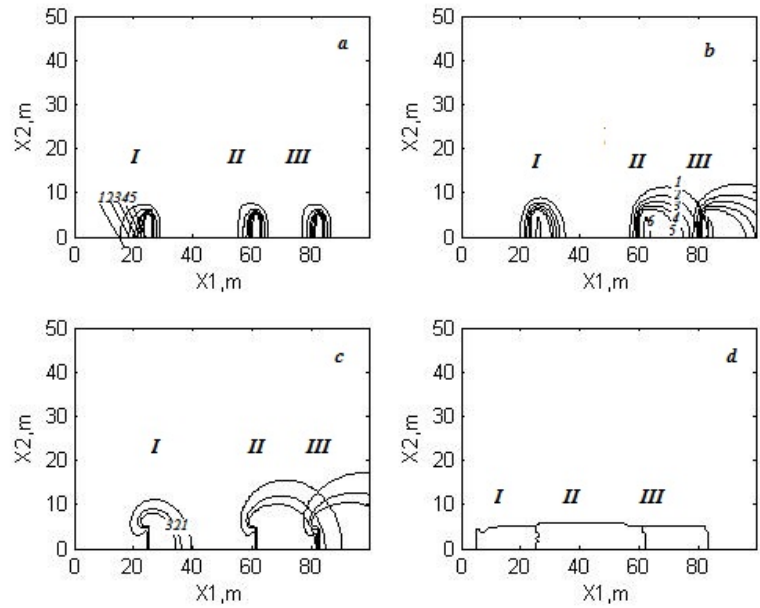

Fig. 7. $V_{\mathrm{e}}=5 \mathrm{~m} / \mathrm{s} ; \mathrm{z}=0.2 \mathrm{~kg} / \mathrm{m}^{3}, W=0.7$.
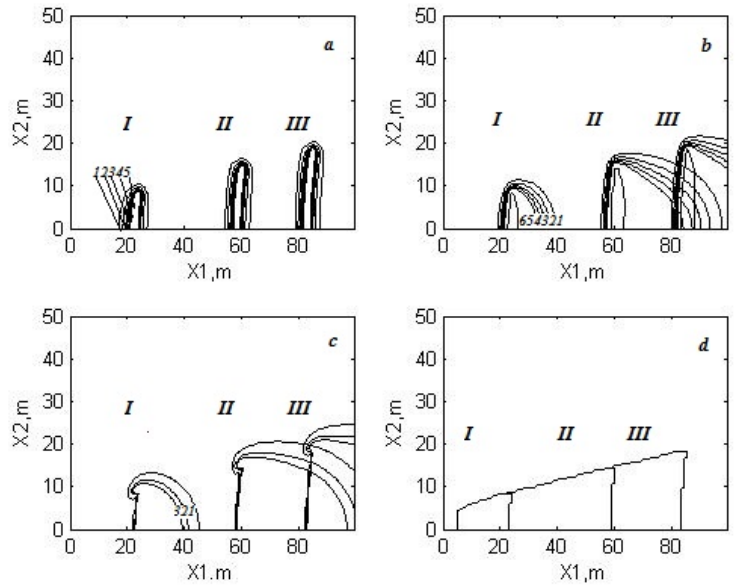

Fig. $8 . V_{e}=5 \mathrm{~m} / \mathrm{s} ; \mathrm{z}=0.4 \mathrm{~kg} / \mathrm{m}^{3}, W=0.6$.
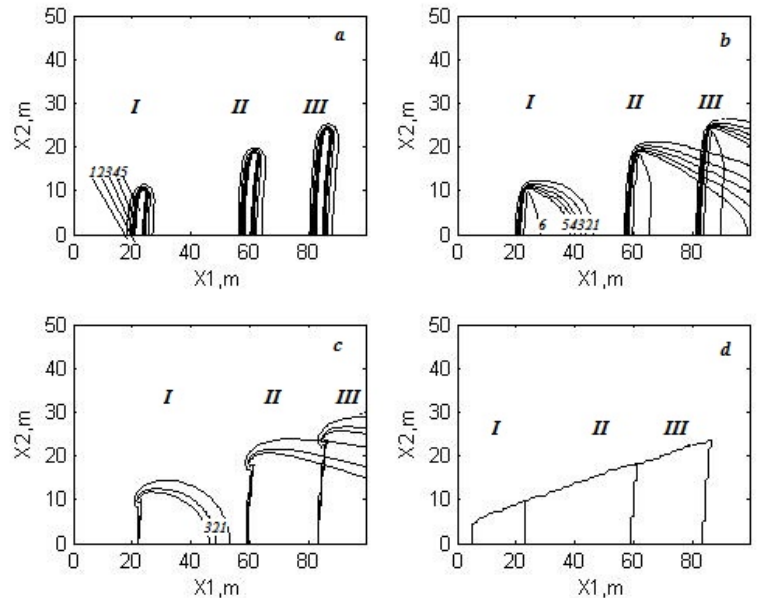

Fig. $9 . V_{e}=5 \mathrm{~m} / \mathrm{s} ; \mathrm{z}=0.6 \mathrm{~kg} / \mathrm{m}^{3}, W=0.6$. 


\section{CONCLUSION}

Results of calculation give an opportunity to describe the different conditions of the forest fire spread taking account different weather conditions and state of forest combustible materials, which allows applying the given model for prediction and preventing fires. It overestimates the rate of crown forest fire spread that depends on crown properties: bulk density, moisture content of forest fuel, wind velocity and etc. The model proposed here gives a detailed picture of the change in the temperature and component concentration fields with time, and determine as well as the influence of different conditions on the crown forest fire spreading for the different state of forest combustible materials.

\section{REFERENCES}

[1] C.E. Van Wagner, Conditions for the start and spread of crown fire. Canadian Journal of Forest Research. 7, 1977, pp. 23-34.

[2] M.E. Alexander, Crown fire thresholds in exotic pine plantations of Australasia. PhD thesis, Department of Forestry, Australian National University, 1998.

[3] C.E. Van Wagner, Prediction of crown fire behavior in conifer stands. In '10th conference on fire and forest meteorology'. Ottawa, Ontario. (Eds D. C. MacIver, H. Auld and R. Whitewood), 1989, pp. 207-212.

[4] G. Xanthopoulos, Development of a wildland crown fire initiation model. PhD thesis, University of Montana, 1990.

[5] R.C. Rothermel, Crown fire analysis and interpretation. In 11th International conference on fire and forest meteorology. Missoula, Montana, USA, 1991.

[6] M.G. Cruz, et al, Predicting crown fire behavior to support forest fire management decision-making. In 'IV International conference on forest fire research'. Luso-Coimbra, Portugal. (Ed. D. X. Viegas), 11 [CD-ROM]. (Millpress) 2002.

[7] F.A. Albini, et al, Modeling ignition and burning rate of large woody natural fuels. Int. Journal of Wildland fire. 5, 1995, pp. 81-91.

[8] J.H. Scott et al, Assessing crown fire potential by linking models of surface and crown fire behavior. USDA Forest Service, Rocky Mountain Forest and Range Experiment Station. Fort Collins: RMRS-RP-29, (Colorado, USA) 2001.

[9] A.M. Grishin, Mathematical Modeling Forest Fire and New Methods Fighting Them. Tomsk, Publishing House of Tomsk University, Russia. 1997.

[10] A.M. Grishin, V.A. Perminov, Mathematical modeling of the ignition of tree crowns. Combustion, Explosion, and Shock Waves, 34, 1998, pp. 378-386.

[11] V.A. Perminov, Mathematical Modeling of Crown and Mass Forest Fires Initiation With the Allowance for the Radiative Convective Heat and Mass Transfer and Two Temperatures of Medium, Ph.D Thesis, Tomsk State University, Russia, 1995.
[12] V.A. Perminov, Mathematical modeling of crown forest fire initiation. In 'III International conference on forest fire research and 14th conference on fire and forest meteorology'. Luso, Portugal. (Ed. D.X.Viegas), 1998, pp.419-431.

[13] E.V. Konev, The physical foundation of vegetative materials combustion.. Novosibirsk, Nauka, Russia. 1977.

[14] D. Morvan, J.L. Dupuy, Modeling of fire spread through a forest fuel bed using a multiphase formulation. Combustion and Flame, 127 (2001) 1981-1994.

[15] D. Morvan, J.L. Dupuy, Modeling the propagation of wildfire through a Mediterranean shrub using a multiphase formulation, Combustion and Flame, 138, 2004, pp.199-210.

[16] S.V. Patankar, Numerical Heat Transfer and Fluid Flow, New York, Hemisphere Publishing Corporation, 1981.

\section{Creative Commons Attribution License 4.0 (Attribution 4.0 International, CC BY 4.0)}

This article is published under the terms of the Creative Commons Attribution License 4.0 https://creativecommons.org/licenses/by/4.0/deed.en_US 